\title{
Design and Simulation of Workshop Layout Reconstruction Based on Flexsim and Multi-objective Genetic Algorithm
}

\author{
Jiawei Xiong ${ }^{1,2}$, Shuhai Fan $^{1,2, *}$, Siyu Chen ${ }^{1}$ and Wenqian Zhang ${ }^{1}$ \\ ${ }^{1}$ Nanjing Tech University, Department of Industrial Engineering, Nanjing, China \\ ${ }^{2}$ MIT Quality Information Program “Data Quality \& Info Security” Lab, Cambridge, MA, USA \\ ${ }^{*}$ Corresponding author
}

\begin{abstract}
Research the problem of dynamic layout of equipment with multiple constraints in reconfigurable workshop. The logistics transport and restructuring of the layout cost as an optimization target. Transforming the dynamic layout problem into a static layout problem in single production cycle. Utilizing the theory of virtual manufacturing and computer simulation to construct a multi - constrained optimization model for dynamic layout of multiple devices with different equipments' acreage. Adopting new genetic algorithm with multi - fitness function and random competition roulette selection strategy to this NP problem that overcomed the shortcomings of a single fitness function that does not guarantee optimal optimization and can not provide multiple strategies for production risk decisions. This approach improved the convergence rate and obtained varieties of feasible solutions from multi-angle model. The effectiveness of the method is verified by examples and Flexsim simulation.
\end{abstract}

Keywords-reconfigurable workshop; equipment dynamic layout; virtual manufacturing; multi - fitness construction genetic algorithm; random competition roulette; flexsim

\section{INTRODUCTION}

With the rise of mass customization production model and the maturity and development of computer integrated manufacturing network, manufacturing enterprises also will be miniaturized and flexible, and the workshop-level production system has also undergone profound changes. The design of the workshop processing system is also pre-operational and intelligent, so the layout of the reconfigurable workshop under the virtual manufacturing framework becomes the research focus of the manufacturing industry.

\section{RECONFIGURABLE WORKSHOP LAYOUT}

\section{A. Reconfigurable Layout}

Reconfigurable workshop floor layout is a branch concept of reconfigurable manufacturing. Reconfigurable workshop layout refers to the processing plant can be based on the production plan flexible and efficient combination of the processing equipment layout. When the new product and processing technology put into operation, it can according to the characteristics of production tasks, in the original layout of the environment efficient, low-cost update shop layout to meet the changing production needs. Reconfigurable shop layout should adhere to the following principles: process, economy, sustainability, safety.

\section{B. Workshop Restructuring Layout Problem Constraints}

The workshop layout problem has always been a very complicated problem. The constraints of the general conditions are: the layout of the equipment placed space; the layout of the equipment; equipment between the distance constraints; product processing process sequence; large equipment placed immovable restrictions and so on. At the same time, the workshop reconfigurable layout also needs to take into account the cost of the layout and the cost of the equipment location adjustment, which makes the reconstruction cost as small as possible. At the same time, the new layout should also make the new layout of the logistics cost less than the original logistics costs.

\section{DESIGN OF RECONFIGURABLE WORKSHOP LAYOUT MODEL}

\section{A. Description of the Relevant Parameters}

Combined with the reconfigurable workshop layout problem model design solution and the constraints, the following parameters are described. There are $n$ device units in the layout space of the workshop. The relevant parameters are as follows:

$\mathrm{L}$ is the available space length;

$\mathrm{H}$ is the available space width;

$\mathrm{M}$ for the total equipment set, $\mathrm{M}=\left\{m_{1}, m_{2}, \cdots, m_{n}\right\}$;

$r_{i j}$ is the handling fee per unit of material per unit distance between device $\mathrm{i}$ and device $\mathrm{j}$;

$R_{i j}$ is the matrix of transport expense;

$$
R_{i j}=\left[\begin{array}{ccc}
r_{11} & \cdots & r_{1 n} \\
\vdots & \ddots & \vdots \\
r_{n 1} & \cdots & r_{n n}
\end{array}\right]
$$

$s_{i j}$ is the transport frequency between device $\mathrm{i}$ and device $\mathrm{j}$; $S_{i j}$ is the transport frequency matrix between devices; 


$$
S_{i j}=\left[\begin{array}{ccc}
S_{11} & \cdots & s_{1 n} \\
\vdots & \ddots & \vdots \\
s_{n 1} & \cdots & s_{n n}
\end{array}\right]
$$

$h_{i j}$ is the minimum lateral distance requirement between device $\mathrm{i}$ and device $\mathrm{j}$;

$H_{i j}$ is the minimum lateral distance requirement matrix between devices;

$$
H_{i j}=\left[\begin{array}{ccc}
h_{11} & \cdots & h_{1 n} \\
\vdots & \ddots & \vdots \\
h_{n 1} & \cdots & h_{n n}
\end{array}\right]
$$

$H_{i 0}$ is the minimum lateral spacing limit for device $\mathrm{i}$ and shop floor boundaries;

$\left(x_{i}, y_{i}\right)$ is the coordinates of device $\mathrm{i}$;

$D_{i j}$ is the distance between the equipments, this paper will define two models of two logistics distance, as shown in figure 1 :

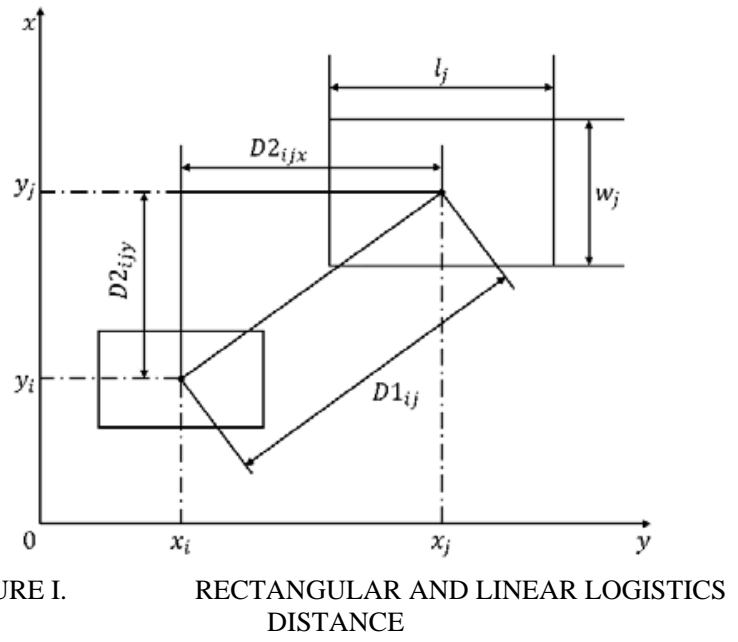

Rectangle distance method:

$$
D_{i j 1}=\left|x_{i}-x_{j}\right|+\left|y_{i}-y_{j}\right|
$$

Straight line distance method:

$$
D_{i j 2}=\sqrt{\left(x_{i}-x_{j}\right)^{2}+\left(y_{i}-y_{j}\right)^{2}}
$$

$D_{i j}$ is the transport distance matrix between devices:

$$
D_{i j}=\left[\begin{array}{ccc}
d_{11} & \cdots & d_{1 n} \\
\vdots & \ddots & \vdots \\
d_{n 1} & \cdots & d_{n n}
\end{array}\right]
$$

$l_{i}$ is the length of device $\mathrm{i}$; $w_{i}$ is the width of device $\mathrm{i}$;

The above parameters are shown in the figure II.

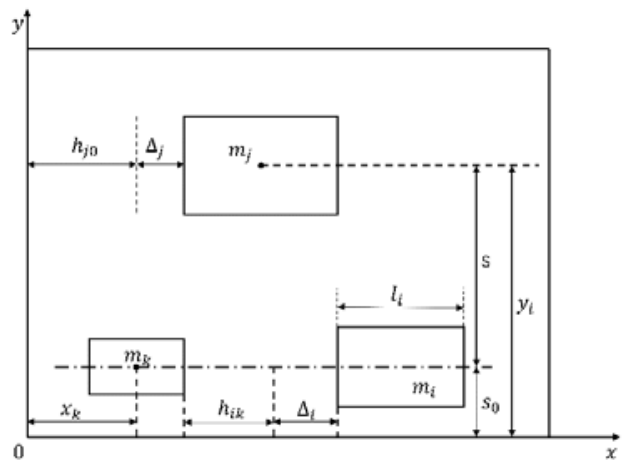

FIGURE II.

DEVICE LAYOUT PARAMETERS, REFERENCE LINES, AND DECISION VARIABLES

B. Mathematical Model Design of Reconfigurable Workshop Layout

1) Minimum logistics cost target: Taking the cost of logistics between each device as main optimization function, the derivation process is as follows:

$$
\begin{gathered}
C 1_{\min }(R, S, D)=\min \sum_{i=1}^{n} \sum_{j=1}^{n} R_{i j} S_{i j} D_{i j} \\
=\min \sum_{i=1}^{n} \sum_{j=1}^{n} R_{i j} S_{i j} \sqrt{\left(x_{i}-x_{j}\right)^{2}+\left(y_{i}-y_{j}\right)^{2}}
\end{gathered}
$$

The objective function of the total logistics cost is:

$$
\min \sum_{i=1}^{n} \sum_{j=1}^{n} R_{i j} S_{i j} \sqrt{\left(x_{i}-x_{j}\right)^{2}+\left(y_{i}-y_{j}\right)^{2}}
$$

$$
C 2_{\min }(R, S, D)=\min \sum_{i=1}^{n} \sum_{j=1}^{n} R_{i j} S_{i j}\left(\left|x_{i}-x_{j}\right|+\left|y_{i}-y_{j}\right|\right)
$$

2) Reconfigurable Constraints: For the reconfigurable equipment layout workshop, the initial placement of the equipment can be carried out twice or even multiple rehandling, is an important indicator of reconfigurability.

Coordinates and boundary constraints:

For $i, j \in\{1,2, \cdots, n\}$

$$
\begin{aligned}
& \left|x_{i}-x_{j}\right| \geq \frac{l_{i}+l_{j}}{2}+h x_{i j} \\
& \left|y_{i}-y_{j}\right| \geq \frac{w_{i}+w_{j}}{2}+h y_{i j}
\end{aligned}
$$


The range $\Delta_{i}$ is the distance between the device $\mathrm{i}$ and the device $\mathrm{i}-1$, that is, the residual distance outside the minimum distance requirement $h_{i j}$ of the device, which is in the range [0, 1.7]; Set the first line of equipment and workshop under the border distance $s_{0}$, the establishment of the following formula:

Device i abscissa:

$$
\begin{array}{r}
x_{i}=x_{k}+\frac{\left(l_{i}+l_{k}\right)}{2}+h_{i k}+\Delta_{i} \\
=h_{k 0}+\Delta_{k}+\frac{\left(l_{i}+2 l_{k}\right)}{2}+h_{i k}+\Delta_{i}
\end{array}
$$

Equipment i Vertical:

$$
y_{i}=(k-1) s+s_{0}
$$

Device independent constraint

In order to meet the requirements of the device does not coincide, the abscissa and the ordinate of the constraints:

$$
\begin{gathered}
\left|x_{i}-x_{j}\right| \geq\left[\frac{\left(l_{i}+l_{j}\right)}{2}+h_{i j}\right] z_{i k} z_{j k} \\
i, j=1,2, \cdots, n \\
z_{i k}=\left\{\begin{array}{cc}
1, \text { device } i \text { in row } \mathrm{k} \\
0, & \text { other }
\end{array} \quad \text { in } 1,2, \cdots, n ; k=1,2, \cdots, m\right.
\end{gathered}
$$

At the same time, a device can only be placed once:

$$
\begin{gathered}
\sum_{k=1}^{m} z_{i k}=1, i=1,2, \cdots, n x_{i}, y_{i} \geq 0, \\
\Delta_{i} \geq 0, \quad i=1,2, \cdots, n
\end{gathered}
$$

\section{IMPLEMENTATION OF LAYOUT ALGORITHM BASED ON GENETIC ALGORITHM}

Encoding, encoding using the device number and equipment net spacing mixed real number coding:

$$
\left[\left\{m_{1}, m_{2}, \cdots, m_{n}\right\},\left\{\Delta_{1}, \Delta_{2}, \cdots, \Delta_{n}\right\}\right]
$$

$m_{i}$ represents the device number; $\Delta_{i}$ represents the net spacing.

Randomly generate the initial population.

Create a penalty function $P_{k}$ :

$$
P_{k}=\left\{\begin{array}{lr}
0, s_{0}+(m-1) s \leq H \\
T, & \text { other }
\end{array}\right.
$$

$H$ is the maximum width of the workshop; $P_{k}$ is the ordinate beyond the penalty term; $T$ is a positive and large value of the penalty value, according to different circumstances generally take $500 \sim 10000$.

Fitness function, according to two definitions for the physical distance can be identified two fitness functions FitnessfuncA and FitnessfuncB.

Center point straight-line distance fitness function:

$$
\text { Fitnessfunc } A=1 /\left(C 1_{\min }+P_{k}\right)=
$$

$$
1 /\left(\min \sum_{i=1}^{n} \sum_{j=1}^{n} R_{i j} S_{i j} \sqrt{\left(x_{i}-x_{j}\right)^{2}+\left(y_{i}-y_{j}\right)^{2}}+P_{k}\right)
$$

Rectangular distance fitness function:

$$
\text { FitnessfuncB }=1 /\left(C 2_{\min }+P_{k}\right)=
$$

$$
1 /\left(\min \sum_{i=1}^{n} \sum_{j=1}^{n} R_{i j} S_{i j}\left(\left|x_{i}-x_{j}\right|+\left|y_{i}-y_{j}\right|\right)+P_{k}\right)
$$

Selection using the Stochastic Tournament-Roulette Wheel Selection. This is a putative random sampling method. This essay adds random competition operation into standard selection, that is, each time the use of roulette operation when the selection of a pair of individuals, so that the two individuals compete alone, leaving the high fitness of individuals, so the cycle, until the election full.

Crossover, due to the use of two sets of data encoding, so the use Part-Mapping Crossover on the device serial number chromosome cross; Arithmetic cross is used to deal with net spacing chromosomes.

Part of the mapping cross, randomly selected two cross points, the two points need to cross the part of the chromosome one by one. Replacing the duplicate symbols at the same time. 


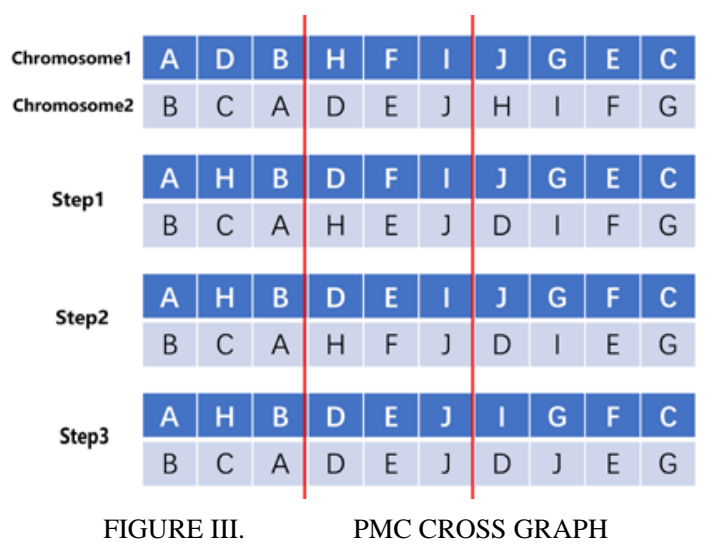

Assuming that two individuals $X_{A}^{\prime}, X_{B}^{\prime}$ are subjected to an arithmetic cross, the new individual is:

$$
\left\{\begin{array}{l}
X_{A}^{t+1}=a X_{B}^{t}+(1-a) X_{A}^{t} \\
X_{B}^{t+1}=a X_{A}^{t}+(1-a) X_{B}^{t}
\end{array}\right.
$$

Since the serial number of the device is encoded as an integer, only the net spacing of the device is mutated. The net spacing chromosome is:

$$
\left[\cdots,\left\{\Delta_{1}, \Delta_{2}, \cdots, \Delta_{i}, \cdots, \Delta_{n}\right\}\right]
$$

\section{CALCUlation EXAMPLES STUDY}

\section{A. Standard Calculation Example}

On the basis of a series of experiments, the parameters of the algorithm are set as follows: population number nchr $=50$, genetic algebra $\mathrm{G}=400$, crossover probability $\mathrm{pc}=0.65$, mutation probability $\mathrm{pm}=0.1$, large penalty value $\mathrm{T}=1000, \mathrm{r}$ $=12$.

In a $11 \mathrm{~m}$ long, $9 \mathrm{~m}$ wide workshop to set up 12 sets of equipment, device size, $R_{i j}, S_{i j}, H_{i j}$ and $H_{i 0}$ are given.

TABLE I. DEVICE SIZE

\begin{tabular}{|l|l|l|l|l|l|}
\hline Device1 & Device2 & Device3 & Device4 & Device5 & Device6 \\
\hline $0.5 * 0.4$ & $1.7 * 0.8$ & $0.8^{*} 0.8$ & $0.7 * 0.7$ & $1 * 0.8$ & $0.9 *^{*} 0.4$ \\
\hline Device7 & Device8 & Device9 & Device10 & Device11 & Device12 \\
\hline $0.8 * 0.8$ & $1.2 * 1$ & $0.5 * 0.8$ & $1.4 * 0.8$ & $1.2 * 0.6$ & $1.4 * 0.8$ \\
\hline
\end{tabular}

In order to shorten the optimization process, a set of initial feasible solutions are obtained with SLP [2 85411173129 106 ] to replace the initial solution.

Optimize this example 15 times and find the best result. The optimal chromosome of the rectangular solution is [(5 121 4112810369 7), (0.2975 0.09940 .03240 .69370 .1755 $\left.\begin{array}{llllllll}0.0449 & 0.0743 & 0.8577 & 0.0948 & 0.1176 & 0.1234 & 0.0673)\end{array}\right]$, the algebra of the optimal solution is 187 generation, the optimal solution is 3427 .

Similarly, the optimal chromosome of the straight-line

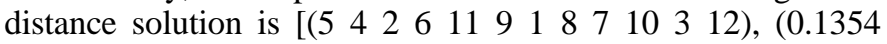
$\begin{array}{llllllll}0.2386 & 0.2521 & 0.2586 & 0.9279 & 0.1466 & 0.1268 & 0.1644 & 0.0221\end{array}$
0.05350 .00600 .0165 )], the algebra of the optimal solution is 127 generation, the optimal solution is 2840 .

It follows that the straight-line distance method can go beyond the general rectangular distance method in terms of convergence speed and accuracy.

\section{B. Example Calculation and Flexsim Simulation}

A factory will establish a new manual operation of the assembly plant, empty space size is $25 \mathrm{~m} \times 15 \mathrm{~m}$, the need for nine processing units for the layout. Population number nchr = 50 , genetic algebra $G=400$, crossover probability $p c=0.65$, mutation probability $\mathrm{pm}=0.1$, large penalty value $\mathrm{T}=5000$, variation interval $r=9$. Device line spacing of $4 \mathrm{~m}$; line 1 equipment ordinate 2.5 .

Calculating the example and the resulting solution is as follows: [1x4 double] [1x4 double] [3]; [5 21946778 3]; The device coordinates are as follows: $(9.9,2.5)(5.7,2.5)(2.9,10.5)$ $(2.5,6.5)(1.4,2.5)(7.7,6.5)(12.7,6.5)(16.8,6.5)(16.1,2.5)$. The evolution of the target values and genetic algebra is shown in the figure:

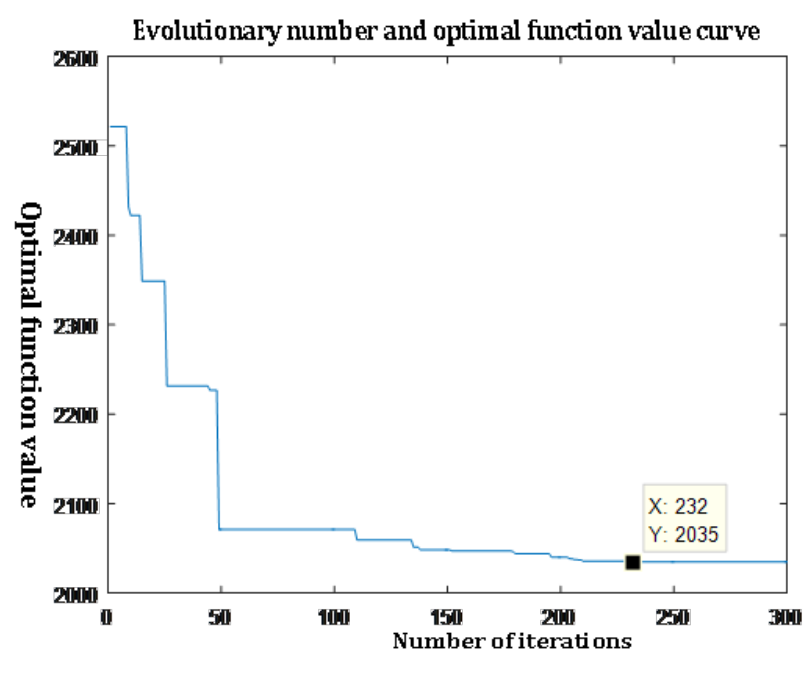

FIGURE IV.

CONVERGENCE ITERATIVE GRAPH

After determining the coordinates of the device, use Flexsim to simulate the obtained data and get the simulated three-dimensional layout. The effectiveness of the method is further verified by simulation. FlexSim simulation results are shown below:

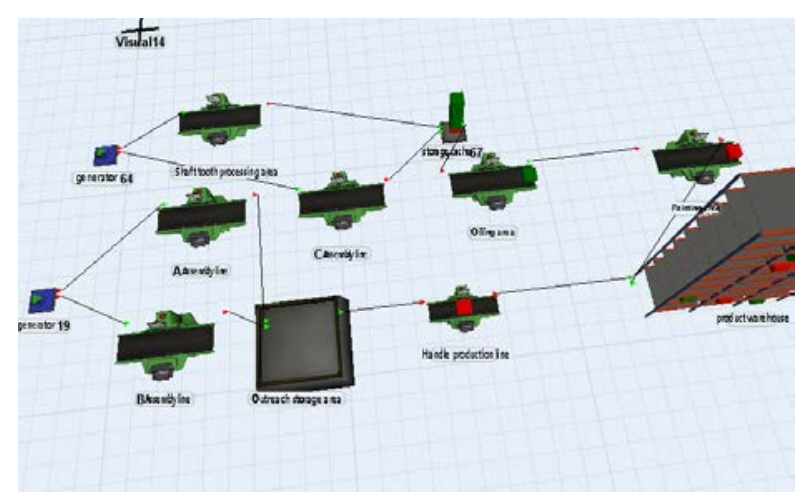

FIGURE V. FLEXSIM LAYOUT SIMULATION 


\section{CONCLUSION}

Using the dual fitness function of genetic algorithm, the choice of two logistics distance can not only bring faster optimization speed, while two different orientation of the decision-making program for enterprise decision makers to bring diversity.

Using the large number of punctuation values and automatic line breaks strategy; cleverly meet the equipment for the size of the site length and width of the two-dimensional constraints.

Improved stochastic tournament-roulette wheel selection select a pair of individuals to compete separately and then put into the mating pool. Reduce the error to ensure a high degree of fitness of the individual selected rate and improve the accuracy of the algorithm.

Based on virtual manufacturing and computer integrated manufacturing principles. The device is pre-arranged using a computer algorithm. And then use Flexsim software to simulate the results of the layout, not only to verify the results of genetic algorithms, but also makes the simulation results more intuitive for future adjustments.

\section{ACKNOWLEDGMENT}

This work was supported by National Natural Science Foundation of China (Grant No. 71671089,71171110, 71701092, 71701093).

\section{REFERENCES}

[1] Chunyan Xia, Shuhai Fan, Yuqian Wang, "Study on Reconfiguration Design of Mixed - flow Production Workshop,” J. Mechanical Design and Manufacture. China, vol. 54, pp. 263-265, 2012.

[2] Xuewen Huang, Xiaotong Zhang, Yaqing Ai, "Application of ant colony algorithm to multi - processing route flexible shop scheduling problem," J. Computer Integrated Manufacturing System. China, vol. 23, pp. 1-19, 2017.

[3] Jin Wang, Shuhai Fan, "Study on Layout of Mixed Flow Production Workshop,” J. Journal of Mechanical Design and Manufacture. China, vol. 47, pp. 252-253, 2010.

[4] Junzheng Huang, Aiping Li, Ming Lei, "Dynamic Layout of Multi target Equipment Based on NSGA II,” J. Journal of China Construction Machinery Corporation. China, vol. 11, pp. 1-6, 2014.

[5] Juan M. Palomo-Romero, Lorenzo Salas-Morera, Laura GarcíaHernández, "An island model genetic algorithm for unequal area facility layout problems,” J. Expert Systems with Applications. China, vol. 68, pp. 151-162, 2017.

[6] Yuanyuan Guo, Qian Wang, Feng Liang. "Design of Workshop Layout Based on Particle Swarm Optimization," J. Computer Integrated Manufacturing Systems. China, vol. 18, pp. 2476-2484, 2012.

[7] Dingyi Wang, Liya Wang. "Application of Improved Genetic Algorithm in Production Shop Layout,” J. Computer Engineering and Applications. China, vol. 41, pp. 190-192, 2005.

[8] Ioanna N.Papadaki, Athanasios P.Chassiakos. "Multi-objective Construction Site Layout Planning Using Genetic Algorithms," J. Procedia Engineering. China,vol. 164, pp. 20-27, 2016.

[9] Huanlin Liu, Xin Wang, Yong Chen, Deqian Kong, Peijie Xia. "Optimization lighting layout based on gene density improved genetic algorithm for indoor visible light communications," J. Elsevier B.V. China, vol. 390, pp. 76-81, 2017.

[10] Jose Antonio Diego-Mas, Rocio Poveda-Bautista, Diana Garzon-Leal. "Using RGB-D sensors and evolutionary algorithms for the optimization of workstation layouts,” J. Applied Ergonomics. China, vol. 65, pp. 530540, 2017.

[11] S. Freitas, F. Serra, M.C. Brito. "Pv layout optimization: String tiling using a multi-objective genetic algorithm,” J. Solar Energy. China, vol. 118, pp. 562-574, 2015. 